\title{
Bacteriophage tailspike protein based assay to monitor phase variable glucosylations in Salmonella O-antigens
}

\author{
Andreas Schmidt ${ }^{1}$, Wolfgang Rabsch, Nina K. Broeker ${ }^{1}$ and Stefanie Barbirz ${ }^{1 *}$ (D)
}

\begin{abstract}
Background: Non-typhoid Salmonella Typhimurium (S. Typhimurium) accounts for a high number of registered salmonellosis cases, and O-serotyping is one important tool for monitoring epidemiology and spread of the disease. Moreover, variations in glucosylated O-antigens are related to immunogenicity and spread in the host. However, classical autoagglutination tests combined with the analysis of specific genetic markers cannot always reliably register phase variable glucose modifications expressed on Salmonella O-antigens and additional tools to monitor O-antigen glucosylation phenotypes of $S$. Typhimurium would be desirable.

Results: We developed a test for the phase variable O-antigen glucosylation state of $\mathrm{S}$. Typhimurium using the tailspike proteins (TSP) of Salmonella phages 9NA and P22. We used this ELISA like tailspike adsorption (ELITA) assay to analyze a library of 44 Salmonella strains. ELITA was successful in discriminating strains that carried glucose 1-6 linked to the galactose of O-polysaccharide backbone (serotype O1) from non-glucosylated strains. This was shown by O-antigen compositional analyses of the respective strains with mass spectrometry and capillary electrophoresis. The ELITA test worked rapidly in a microtiter plate format and was highly O-antigen specific. Moreover, TSP as probes could also detect glucosylated strains in flow cytometry and distinguish multiphasic cultures differing in their glucosylation state.

Conclusions: Tailspike proteins contain large binding sites with precisely defined specificities and are therefore promising tools to be included in serotyping procedures as rapid serotyping agents in addition to antibodies. In this study, 9NA and P22TSP as probes could specifically distinguish glucosylation phenotypes of Salmonella on microtiter plate assays and in flow cytometry. This opens the possibility for flow sorting of cell populations for subsequent genetic analyses or for monitoring phase variations during large scale O-antigen preparations necessary for vaccine production.
\end{abstract}

Keywords: Salmonella Typhimurium, O-antigen, Tailspike protein, Bacteriophage, Phase variation, O-serotyping, Flow cytometry

Abbreviations: CE-LIF, Capillary electrophoresis with laser-induced fluorescence detection; ELITA, ELISA like tailspike adsorption; HPAEC-PAD, High performance anion exchange with pulsed amperometric detection;

LPS, Lipopolysaccharide; MALDI-MS, Matrix-assisted laser-desorption ionization mass spectrometry; TSP, Tailspike protein.

\footnotetext{
*Correspondence: barbirz@uni-potsdam.de

${ }^{1}$ Physikalische Biochemie, Universität Potsdam, Karl-Liebknecht-Str. 24-25,

Golm 14476, Germany

Full list of author information is available at the end of the article
} 


\section{Background}

Antimicrobial strategies usually focus on bacterial surfaces, for example in classic antibiotics that interfere with cell wall synthesis pathways [1], and also in phage therapy approaches that target the outer membrane for penetration [2]. Moreover, classical immunological diagnostics rely on the recognition of specific surface compounds by antibodies which are described for Salmonellae in the Kauffmann-White-Le Minor typing scheme [3]. It defines, amongst others, the $\mathrm{O}$-antigen, a repetitive polysaccharide of highly diverse carbohydrate composition [4]. In Salmonellae, O-antigens often vary in their glucosylation states [5]. Salmonellae contain up to four gtr operons for glucose transfer controlled by epigenetic phase variation [6] and of bacteriophage origin, adding an additional level of complexity to $\mathrm{O}$-antigen structure which is only partly reflected by the Kauffmann-White-Le Minor scheme [7]. $S$. Typhimurium has the O-serotype O4, O12 and is one of the most commonly isolated strains in non-typhoid Salmonellosis infections. Furthermore, as a consequence of phase variation glucose can be 1-4 linked (serotype O12-2) or 1-6 linked (serotype O1) to the galactose of O-polysaccharide backbone [6-9]. In addition, O-antigen acetylation can occur, which defines the serotype O4,(5) [9]. Serotypes are usually classified by slide agglutination tests with antibodies in combination with analysis of genetic markers [10], but these tests do not always reliably reflect the highly variable glucosylation state of the $S$. Typhimurium O-antigen. For example, phase variable $\mathrm{O}$-antigen glucosylations may influence the evasion of Salmonella from complement [11] or can occur only upon exposure to eukaryotic cells, whereas they may be absent in bacteriologic media [5]. Moreover, glucosylations also protect Salmonella from bacteriophage infection [12] and regulate prophage associated $\mathrm{O}$-antigen variability [13].

Bacteriophage tailspike proteins (TSP) of lipopolysaccharide (LPS) specific phages recognize and cleave the $\mathrm{O}$-antigen to position the phage towards a secondary membrane receptor during infection [14-16]. They possess large carbohydrate interaction sites that bind $\mathrm{O}$-antigen with high specificity [17-19]. TSP are highly thermostable and protease resistant [20]. TSP of bacteriophage 9NA and P22 recognize $S$. Typhimurium and have endorhamnosidase activity to cleave the $\mathrm{O}$-antigen $[16,21]$. In this work we present a methodology using 9NA and P22TSP as probes for the rapid detection of $\mathrm{O}$-antigen glucosylation in $S$. Typhimurium both on microtiter plates and in flow cytometry.

\section{Results}

ELISA-like tailspike adsorption (ELITA) assay

To rapidly select for bacterial cultures binding to bacteriophage tailspike proteins (TSP) we developed a microtiter-plate based assay. For this, the bacteria were adsorbed to the surfaces of the wells. We then used Strep-tag ${ }^{\circledR}$ II-labelled 9NATSP to probe binding to $44 \mathrm{Sal}$ monella strains of the Wernigerode collection and read out the signal with a Strep-Tactin ${ }^{\odot}$ labeled horseradish peroxidase. The resulting ELISA-like tailspike adsorption (ELITA) procedure was highly specific (Fig. 1). 9NATSP recognized strains of O-serogroups O2, O4,(5) and O9. These are the serogroups found in the host range of bacteriophage 9NA [21]. By contrast, no signals were observed for non-host strains and for an E. coli strain defined as the false positive control. The host strain $S$. Typhimurium DB7136 used to propagate bacteriophage 9NA was used to normalize the binding signal and served as false negative control. The test was repeated four times and standard deviation from the independent experiments was less than $10 \%$. In the ELITA test we used a TSP that was mutated to inactivate its enzymatic cleavage of the $\mathrm{O}$-polysaccharide in order to obtain a stable binding signal [16]. If we pre-incubated the bacteria with an enzymatically active TSP, no binding was detectable with the Strep-tag ${ }^{\oplus}$ II-labelled 9NATSP probe afterwards (Fig. 1). This further confirmed that the 9NATSP binding signal in the ELITA was clearly Oantigen specific. However, within the subset of binding strains, 9NATSP showed signal intensities varying from strain to strain. We purified several LPS from strains with different signals in the ELITA assay and did not observe varying chain lengths which might account for different numbers of TSP binding sites and therefore varying ELITA signals (data not shown). Rather, it is plausible that the ELITA test reflected the heterogeneous adsorption behavior of different strains to the microtiterplate surface.

\section{Analysis of O-antigen composition of 9NATSP binding Salmonella strains}

About half of the strains tested were of O-serogroup O4 or $\mathrm{O} 4,(5)$. These $\mathrm{O}$-antigens have the trisaccharide backbone structure $\alpha$-D-Man $p$-1-4- $\alpha$-L-Rhap-1-3- $\alpha-\mathrm{D}-\mathrm{Gal} p$ - $\alpha$ 1-2 [22]. The didesoxyhexose abequose $\alpha-1-3$-linked to mannose determines the serotype $\mathrm{O} 4$, if the abequose is acetylated, the serotype O4,(5) results [9]. We chose several of these strains that showed similar binding signals in ELITA with 9NATSP and examined the molecular composition of the O-antigen. For this, we analyzed oligosaccharides purified from 9NATSP-O-polysaccharide digests with MALDI-MS (Fig. 2 and Table 1). The mass spectra showed peaks corresponding to fragments of two Oantigen repeat units (2RU) as main digestion products in all samples in agreement with precedent studies on $\mathrm{O} 4 \mathrm{li}$ popolysaccharides [16]. For several strains acetylations were detected. Additionally, some strains showed peaks at higher masses corresponding to 2RU fragments with two additional hexoses, for example in case of $S$. Brancaster 


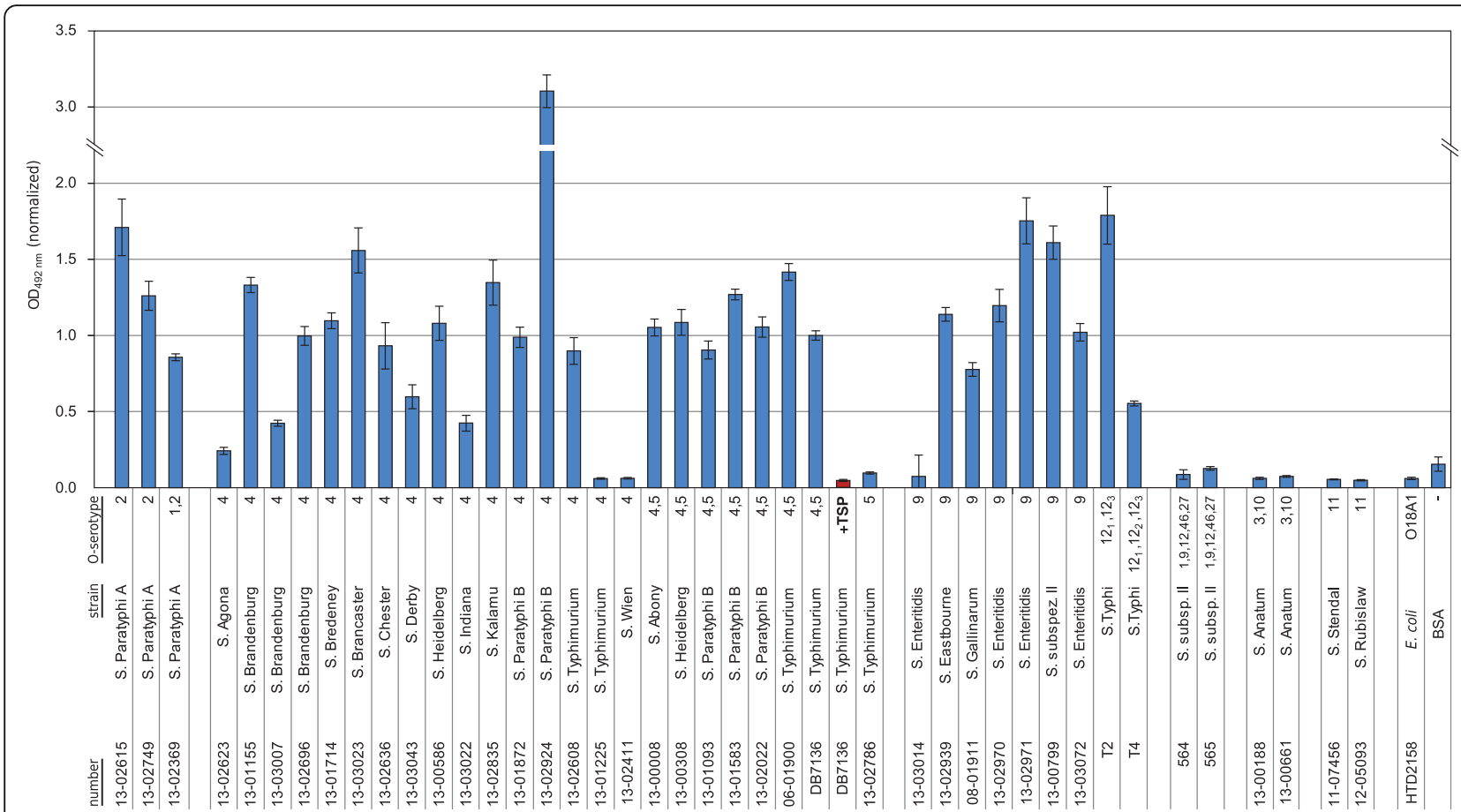

Fig. 1 Analysis of Salmonella strains with ELISA-like tailspike adsorption (ELITA) assay using Strep-tag ${ }^{\oplus} \mid$-labelled 9NATSP as a probe. One sample shows strain DB7136 incubated with 9NATSP wild type prior to ELITA (red bar). Strain E. coli HTD2158 [38] and bovine serum albumin (BSA) were used as a negative control. Numbers refer to the Wernigerode collection ( $c f$. Table 3). Error bars represent standard deviation from four replicate experiments

(Fig. 2b). The analysis of the monosaccharide composition of $S$. Brancaster O-polysaccharide with high performance anion exchange with pulsed amperometric detection (HPAEC-PAD) confirmed the presence of glucose in the $\mathrm{O}$-antigen of this strain (Additional file 1: Figure S1). Glucosylated oligosaccharides were also detected with polysaccharide from $S$. Paratyhi B 2924, from $S$. Kalamu and $S$. Heidelberg 308. By contrast, polysaccharide from strain $S$. Heidelberg 586 only showed non-glycosylated oligosaccharides. From this we conclude that 9NATSP tolerates a glucosylated O-antigen.

\section{Comparison of binding properties of two Salmonella phage TSP with similar host range}

The 44 Salmonella strains analyzed with the 9NATSP probe were subsequently tested in the ELITA assay using a TSP probe from bacteriophage P22 (see Additional file 2: Figure S2 for the full data set). P22TSP is structurally very similar to 9NATSP because both phages 9NA and P22 use their TSP as essential infection organelles for specific host adsorption on a similar host range [16]. In the ELITA assay, P22TSP, like 9NATSP, clearly distinguished all host from non-host strains. However, within a single strain P22TSP and 9NATSP markedly differed in their binding signals. This was distinctly visible in the O4,(5) subgroup, where P22TSP showed higher signals for $S$.
Heidelberg 586, S. Paratyphi B 1872 and S. Agona (Fig. 3). By contrast, P22TSP displayed markedly reduced binding signals for the strains $S$. Brancaster, $S$. Kalamu, $S$. Heidelberg 308 and S. Paratyphi B 2924. These strains all contain glucose in their $\mathrm{O}$-antigens. They were not only impaired in P22TSP binding but also in O-polysaccharide cleavage by P22TSP (Fig. 3). If they were pre-treated with enzymatically active P22TSP, binding was still detectable after this with the 9NATSP probe, showing that the Oantigen had remained intact. This data is in agreement with the fact that P22TSP and 9NATSP differ in their ability to recognize and cleave glucose containing O4polysaccharides [21]. To confirm this we analyzed the products obtained from TSP-mediated cleavage of LPS preparations isolated from different O4,(5) Salmonella strains with capillary electrophoresis (CE) (Fig. 4). Here, the main 2RU cleavage products eluted at later retention time when they contained glucose. 9NATSP and P22TSP both produced octasaccharides from the non-glucosylated strain S. Heidelberg 586. From all other strains that were glucosylated, P22TSP only produced non-glucosylated octasaccharides. By contrast, analyzing 9NATSP LPS cleavage products in $\mathrm{CE}$ showed $2 \mathrm{RU}$ products containing $89 \%$ glucose in $S$. Brancaster, $39 \%$ in $S$. Paratyphi B 2924 and $100 \%$ in S. Heidelberg 308, respectively. 

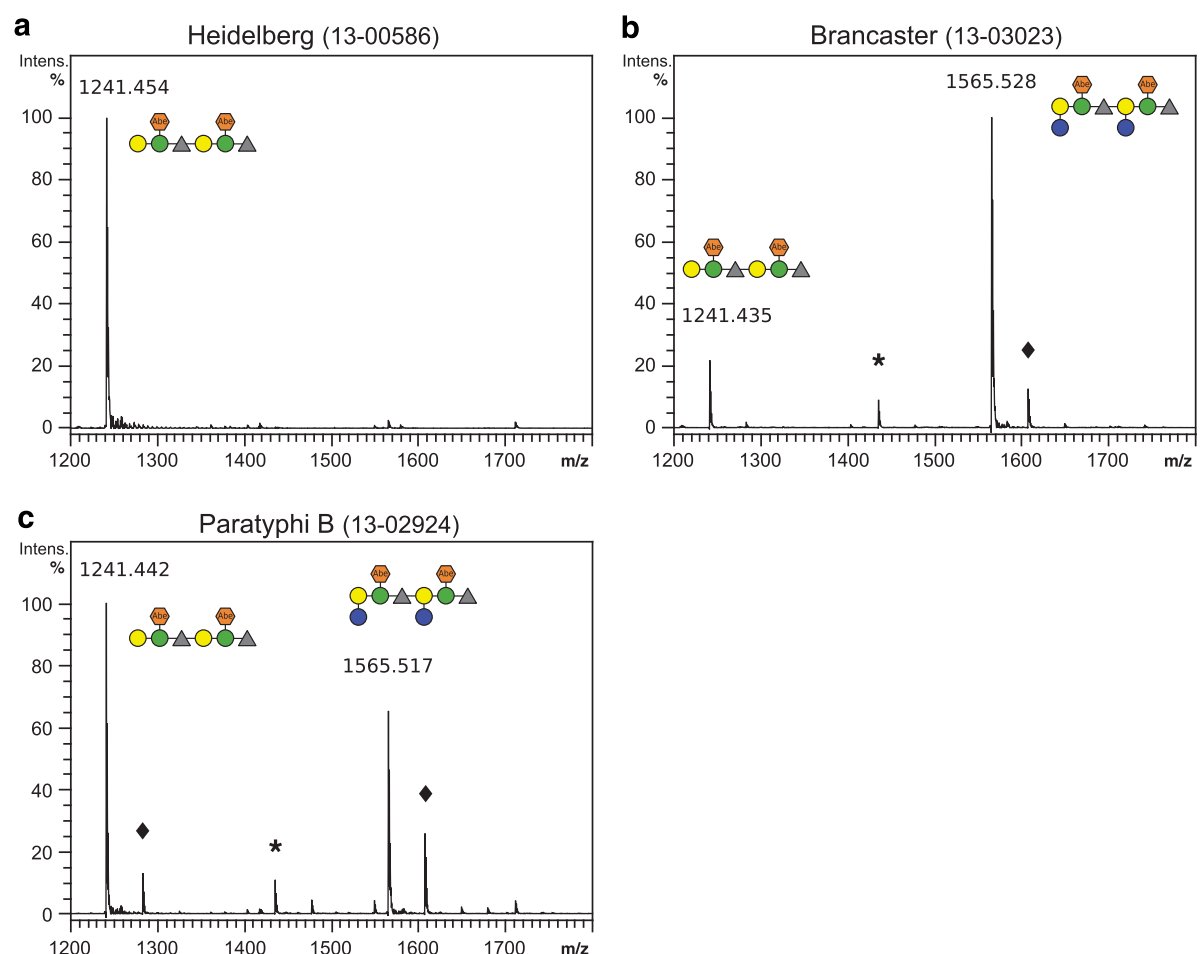

Fig. 2 MALDI-MS of the two repeat unit fraction oligosaccharides obtained from 9NATSP cleavage of polysaccharide isolated from a $S$. Heidelberg 586, b S. Brancaster, and c S. Paratyphi B 2924. Putative oligosaccharide structures are given in CFG notation [42] with abequose symbolized as red hexagon. Asterisks mark peaks that lost abequose, diamonds mark acetylated peaks, respectively. All theoretical and experimental masses are given in Table 1

\section{Flow cytometry analysis of 9NATSP and P22TSP binding to Salmonella}

O-antigen glucosylation can occur as a phenotype during phase variation in Salmonella [6]. We analyzed bacterial cultures with flow cytometry for the occurrence of heterogenous populations [23]. Moreover, the flow cytometry experiment served as a control to exclude any matrix effects in the microtiter plate based ELITA. We incubated bacteria with Strep-tag ${ }^{\circ}$ II-labelled TSP, stained bound TSP with a fluorescent Strep-Tactin ${ }^{\oplus}$-Chromeo488 conjugate and subjected the bacteria to flow cytometry. Binding efficiency was measured as the load of TSP per bacterial particle, as deduced from the increase of Chromeo488 fluorescence (see Additional file 3: Figure S3 for the full data sets). 9NA and P22TSP bound to 69 and $83 \%$ of the cells of the reference strain $S$. Typhimurium DB7136, respectively, but not to the E. coli control (Fig. 5). Both TSP also bound well to the majority of cells of the nonglycosylated strain $S$. Heidelberg 586 which contained even more binding cells than the reference (86 and $94 \%$, respectively). By contrast, the glucose containing strains $S$. Brancaster, S. Kalamu and S. Paratyphi B 2924 displayed reduced binding with P22TSP compared to the signals obtained with 9NATSP, in qualitative agreement with results

Table 1 Monoisotopic calculated and experimental mass analyses from MALDI-MS of two repeat unit (2RU) oligosaccharides obtained from O-antigen polysaccharide enzymatic cleavage with 9NATSP

\begin{tabular}{|c|c|c|c|c|}
\hline \multirow{3}{*}{$\begin{array}{l}\text { Oligosaccharide } \\
\text { composition }^{a}\end{array}$} & \multirow[t]{3}{*}[\mathrm{M}+\mathrm{Na}]{$_{\mathrm{calc}}^{+} / \mathrm{Da}$} & \multicolumn{3}{|l|}{$[\mathrm{M}+\mathrm{Na}]_{\mathrm{exp}}^{+} / \mathrm{Da}$} \\
\hline & & \multicolumn{3}{|l|}{ Salmonella strain } \\
\hline & & Heidelberg 586 & Brancaster & Paratyphi B \\
\hline $2 \mathrm{RU}$ & 1241.45 & 1241.45 & 1241.44 & 1241.44 \\
\hline $2 \mathrm{RU}+2 \mathrm{Glc}-\mathrm{Abe} \mathrm{e}^{\mathrm{b}}$ & 1435.41 & n.d..$^{c}$ & 1435.47 & 1435.44 \\
\hline $2 \mathrm{RU}+2 \mathrm{GlC}$ & 1565.56 & n.d. ${ }^{c}$ & 1565.53 & 1565.52 \\
\hline $2 \mathrm{RU}+2 \mathrm{Gl}+\mathrm{Ac}$ & 1607.57 & n.d. ${ }^{c}$ & 1607.53 & 1607.52 \\
\hline
\end{tabular}

two repeat units of basic repeat a-D-Galp-1-2-a-D-Manp(-a-3-1-Abep)-1-4-a-L-Rhap-1-3- as produced by TSP cleavage [44], Abe: Abequose, Ac: Acetylation b without Abequose

cnot detected 

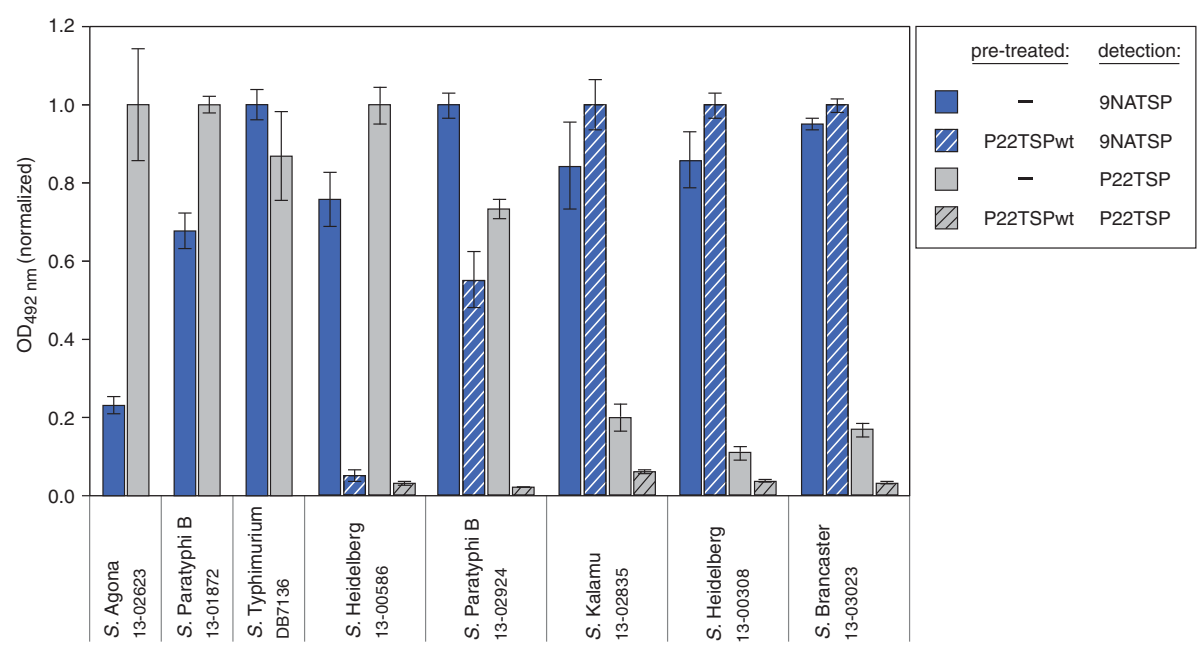

Fig. 3 ELISA-like tailspike adsorption (ELITA) assay with Salmonella strains of serogroup O4,(5) using 9NATSP (blue) or P22TSP (gray) as probes. Crosshatched bars represent data obtained after pre-treatment of immobilized bacteria with $50 \mathrm{\mu g} / \mathrm{ml}$ of catalytically active P22TSP to destroy the O-antigen receptor. Signals were normalized individually for each single strain; error bars represent standard deviation from four replicate experiments.
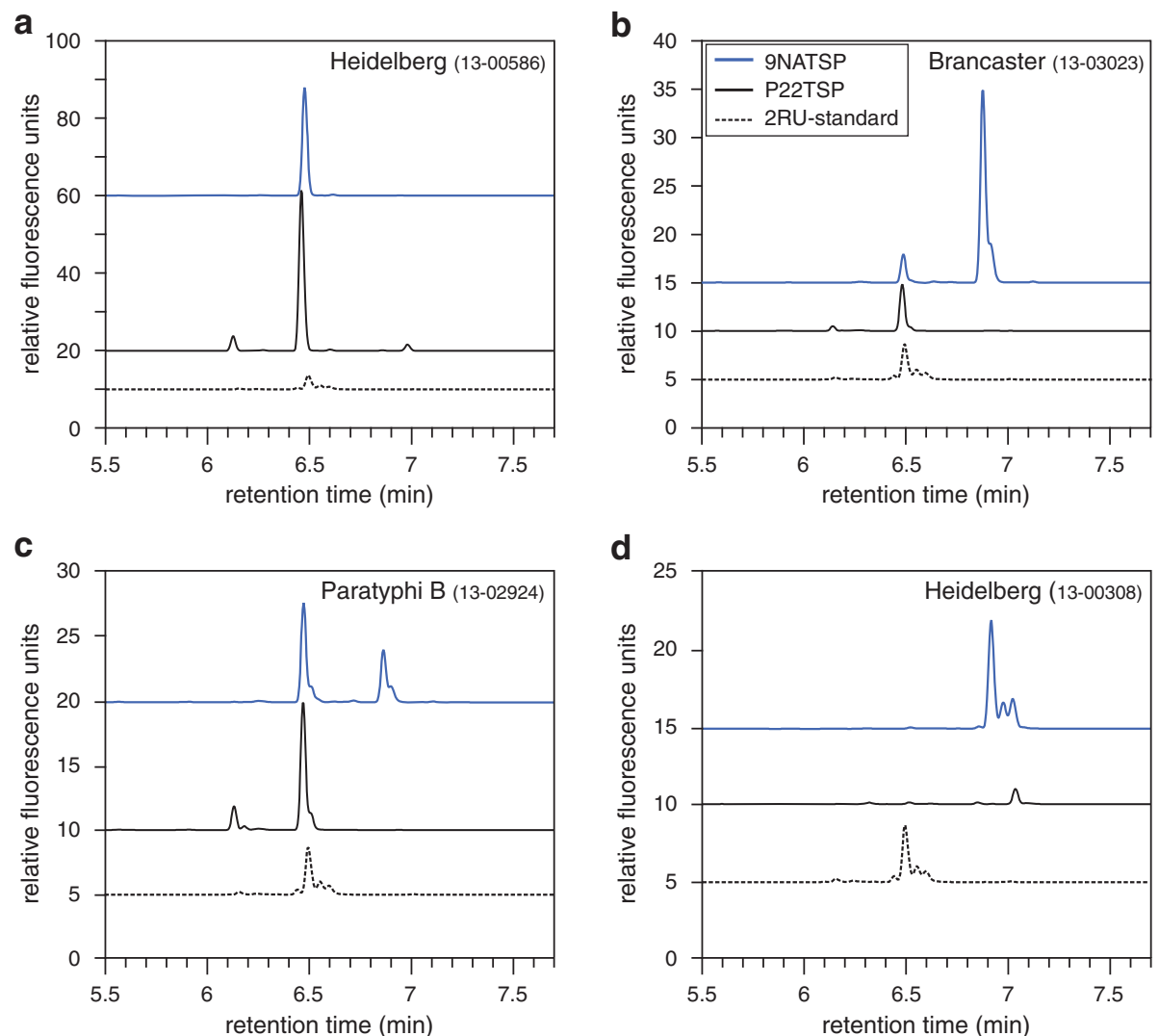

Fig. 4 Analysis of oligosaccharide mixtures obtained from Salmonella LPS after TSP enzymatic cleavage with capillary electrophoresis and laser-induced fluorescence detection (CE-LIF). Profiles from LPS of S. Heidelberg 586 a, S. Brancaster b, S. Paratyphi B 2924 c, S. Heidelberg 308 d shown after treatment with 9NATSP (blue) or P22TSP (black). The CE-LIF profile of a 2RU standard shown as dashed line 

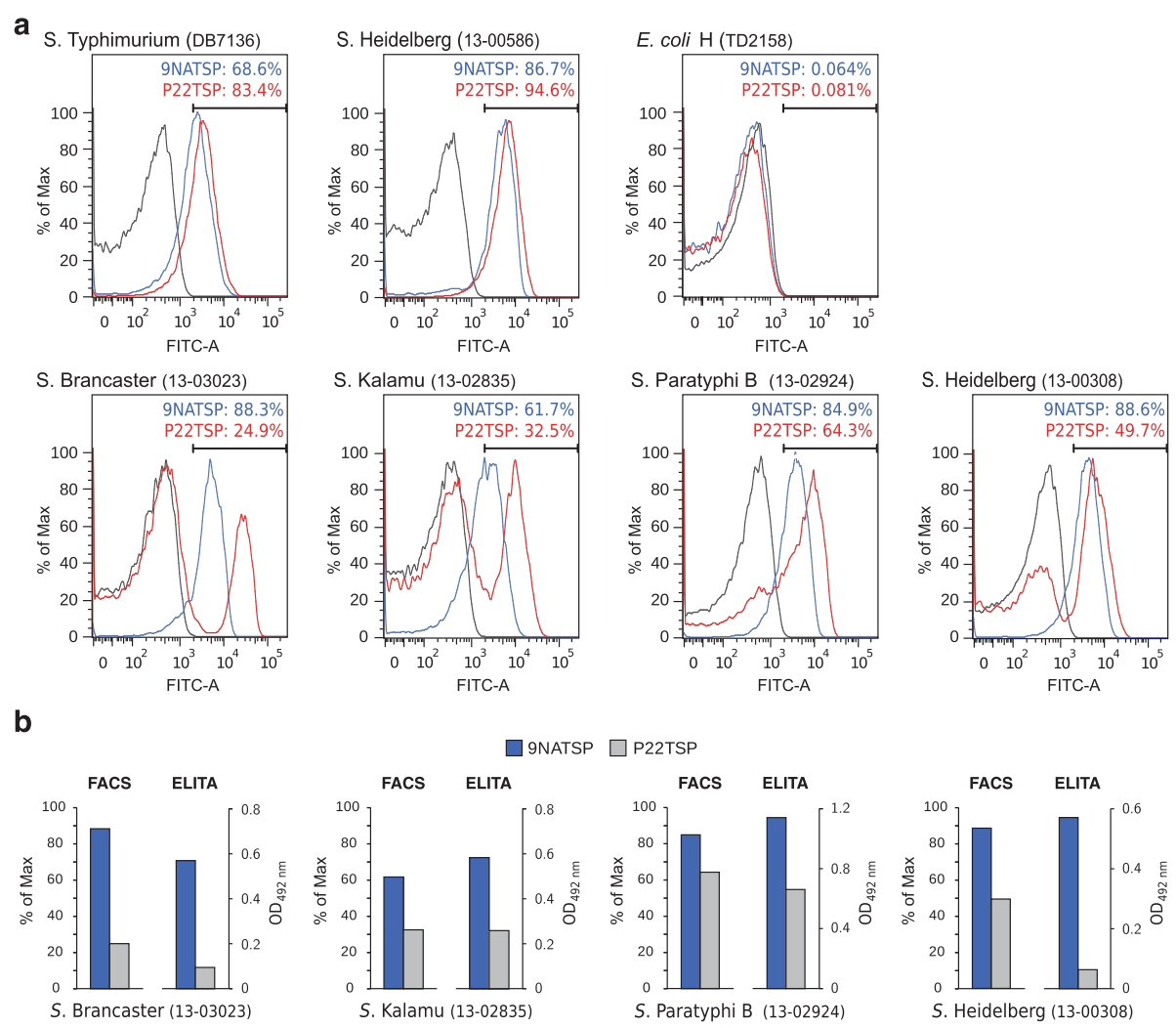

Fig. 5 a Flow cytometry histograms of a representative set of Salmonella strains from serogroup O4,(5) probed with fluorescently tagged TSP. b Qualitative comparison of signal intensities from ELITA assay and flow cytometry

obtained in the ELITA test (Fig. 5). Apparently, P22TSP only bound to the subpopulations of non-glycosylated cells whereas another subpopulation remained unbound. This increased the TSP to cell ratio and resulted in higher signal intensities for the binding population. We conclude that P22TSP, in contrast to 9NATSP could distinguish glucosylated from non-glycosylated subpopulations in these strains. Interestingly, S. Heidelberg 308 showed about $50 \%$ binding to P22TSP and $90 \%$ binding to 9NATSP in flow cytometry. This is contradictory, as this strain was found glucosylated and resistant to P22TSP cleavage (see above). Moreover, $S$. Heidelberg 308 only showed weak binding in the ELITA test. 2RU fragments produced from $S$. Heidelberg 308 by 9 NATSP O-antigen migrated in CE as glucosylated oligosaccharides with additional minor peaks indicating acetylated species. We can only speculate at this point, that cells at mid-logarithmic phase as analyzed in flow cytometry displayed smaller amounts of acetyl or glucosyl groups on their surface than those grown to stationary phase used for LPS preparations and in ELITA. In the flow cytometry experiment several strains tended to aggregate in the presence of TSP but not without TSP, as deduced from the size of detected particles (Additional file 3: Figure S3). This is probably due to the multivalency of the detection system with three Strep-tags ${ }^{\circ}$ II per TSP and four binding sites on the Strep-Tactin ${ }^{\circ}$-Chromeo488 conjugate which results in cross linking of bacteria [24]. Accordingly, no aggregation was observed when adding TSP without tags (data not shown).

\section{Bacteriophage infection assays}

Binding of a TSP to the bacterial surface is an initial step in the bacteriophage infection cycle $[15,16]$. Therefore we tested whether TSP binding observed in the ELITA assay or in flow cytometry correlated with infectivity of bacteriophages 9NA and P22 on the respective strains. We determined efficiency of plating (EOP) in soft agar layers on a subset of Salmonella strains belonging to the serogroup O4,(5) (Table 2). Both phages displayed similar infectivity on the reference host strain DB7136. Moreover, phage 9NA could infect $S$. Paratyphi B 2924, here 9NATSP also was a very good binder ( $c f$. Fig. 3). The tailspike of phage P22 was a good binder on $S$. Heidelberg 586, accordingly the phage could produce a small amount of plaques on this strain $(\mathrm{EOP}<0.1 \%)$. All other strains were resistant $(\mathrm{EOP}<0.001 \%)$ to both phages, notwithstanding that 9NATSP had shown good binding signals on all, and P22TSP on two of the strains tested. Apparently, more criteria must lead to successful 
Table 2 Efficiency of plating (EOP) of bacteriophages P22 and 9NA on Salmonella of serogroup $\mathrm{O4},(5)$

\begin{tabular}{|c|c|c|c|c|}
\hline \multirow[t]{2}{*}{ Salmonella strain } & \multicolumn{2}{|l|}{ 9NA } & \multicolumn{2}{|l|}{ P22 } \\
\hline & $\overline{E O P^{a}}$ & $p f u^{\mathrm{b}} / \mathrm{ml}^{-1}$ & $\overline{E O P}$ & $p f u / m^{-1}$ \\
\hline Typhimurium DB7136 & 1 & $1.80 \times 10^{12}$ & 1 & $5.00 \times 10^{11}$ \\
\hline Paratyphi B 2924 & 0.16 & $2.81 \times 10^{11}$ & - & - \\
\hline Brancaster & - & - & - & - \\
\hline Kalamu & $1.70 \times 10^{-6}$ & $3.06 \times 10^{6}$ & - & - \\
\hline Heidelberg 13-00586 & - & - & $1.14 \times 10^{-3}$ & $5.69 \times 10^{8}$ \\
\hline Heidelberg 13-00308 & - & - & - & - \\
\hline
\end{tabular}

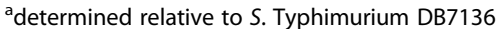

${ }^{b}$ number of plaque forming units (pfu) detected

phage infection than just TSP recognition of the right $\mathrm{O}$-serotype polysaccharide. However, comparing phage plating with TSP binding experiments, TSP binding seems at least necessary albeit not sufficient for successful Salmonella infection by bacteriophages 9NA and P22.

\section{Discussion}

In the present study, TSP from siphovirus 9NA and podovirus P22 were used to monitor the glucosylation state of the Salmonella O-antigen of serotype O4. We propose that in the ELITA test, both TSP could distinguish the glucosylation state of strains due to their different specificities. P22TSP could not bind to 1-6 glucosylated $\mathrm{O}$-antigen, these serogroup O1 strains carry the bacteriophage P22 lysogen, the glucosylation of which prevents phage P22 from infection [25]. By contrast, 9NATSP bound to these strains, which implies that it can specifically bind 1-6 linked glucose. Comparing the O-antigen binding sites of 9NA and P22TSP we propose that in the P22TSP binding site, glucose at this position would probably not fit into the pocket (Fig. 6). By contrast, the binding site of 9NATSP contains a large surface cavity which could probably accommodate 1-6 linked glucose. This further corroborates that 9NATSP is specific for serogroup O1 whereas P22TSP cannot recognize the 1-6 glucose modification [21]. We also found O4 strains where 9NATSP was a weak binder while P22TSP showed a high signal, i.e. for $S$. Agona, S. Brandenburg, S. Chester or $S$. Derby (Additional file 2: Figure S2). Although we have not analyzed the glucose content in $\mathrm{O}$-antigens of these strains we propose that they carry 1-4 glucosylations (serotype O12-2) because analysis of phage endorhamnosidase activities had shown earlier that phage 9NA in contrast to phage P22 was unable to produce oligosaccharides from O12-2 strains [21]. This was further supported by an ELITA test on $S$. Typhi, where P22TSP showed good binding on a O12-2 typed strain whereas 9NATSP showed a weak signal on this strain (Additional file 2: Figure S2). P22TSP can bind to O12-2 O-antigens as shown by crystal structure analysis [26]. The overlay of this structure with the 9NATSP binding site could not show why 9NATSP does not tolerate the 1-4 glucosylation and we are yet to solve a crystal structure to analyze why the architecture of the 9NATSP O-antigen interaction site prevents binding of O12-2 O-antigens. Taken together, P22TSP and 9NATSP are thus useful probes to distinguish O1 from non-O1 Salmonella serogroup O4 strains when employed in a comparative ELITA test (cf. Fig. 3). Moreover, we suggest that they could be employed to distinguish O12-2 positive from O12-2 negative strains, although

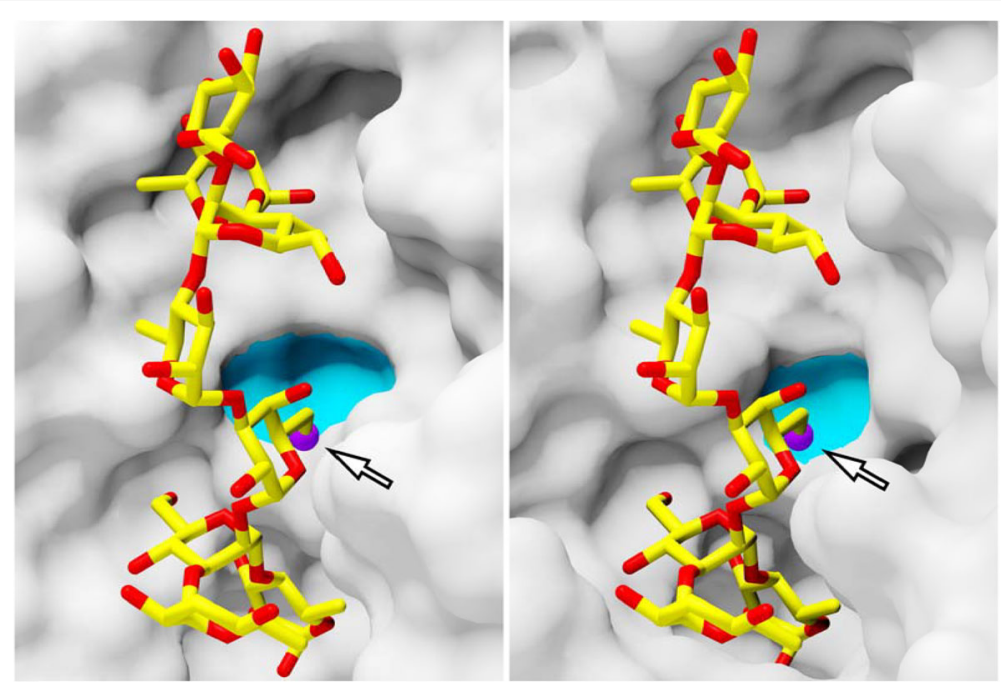

Fig. 6 Comparison of 9NATSP and P22TSP O-antigen binding sites. The O6 of galactose pointing to the protein surface is shown in magenta (see arrow), a putative glucose binding groove in cyan. Right: Crystal structure of P22TSP with 2RU oligosaccharide (pdb: 1tyx) [26]. Left: Crystal structure of 9NATSP (pdb: 3riq) [16]. The 2RU oligosaccharide was positioned in the binding site after 3D alignment with P22TSP (rmsd 2.54 A) using the CEALIGN algorithm implemented in PyMOL [43] 
the molecular details for the lacking interaction with 9NATSP remain to be elucidated.

In the ELITA assay also weak signals occurred for strains that had a glucosylation pattern prohibiting binding to the respective TSP probe. This indicated that heterogeneous cell populations were present on the microtiter plate. Effects related to surface adhesion of bacteria on the plate can be excluded as relative binding efficiencies were similar for the same strains in flow cytometry ( $c f$. Fig. 5). More importantly, flow cytometry using 9NA and P22TSP as probes confirmed the presence of heterogeneous populations in the investigated cultures of serotype O1. In these cases, the size of the populations detected with P22TSP correlated well with the proportions of nonglucosylated O-antigen measured in CE-LIF. 9NA and P22TSP are therefore very interesting tools in flow cytometry to monitor glucosylation phenotypes of Salmonella. For example they could be used to separate cell populations in a flow sorter and further analyze them by sequencing, with PCR techniques [27] or for studying phase variations in succeeding cell generations. Monitoring phase variations is also important for $\mathrm{O}$-antigen structural analysis or to obtain defined precursors for vaccine production. Here, the glucosylation state is closely related to the immunogenicity of the O-antigen [28]. For the preparation of sufficient material, cells have to be grown to high densities [29]. Flow cytometry with TSP probes could be used here to rapidly assess the glucosylation state of cultures. For strain Heidelberg 308 we observed these differences depending on the time of growth. Whereas overnight cultures as used for $\mathrm{O}$-antigen preparation were fully glucosylated and resistant to P22TSP cleavage, mid-log phase cultures analyzed by flow cytometry using the P22TSP probe showed a binding population indicating non-glucosylated species.

Recognition of defined molecular structures also distinguishes TSP from whole phage typing procedures. Type phages usually are not purely $\mathrm{O}$-antigen specific but also require protein or core LPS receptors for broad specificity. Here, successful infection and replication are a consequence of the multitude of recognition, membrane penetration, replication and lysis events that bacteriophages pass through during their life cycle. This makes phage typing a specific tool for classification of Salmonella strains as plaque formation is tightly coupled to the biochemical state of the bacterial cell [30]. However, phage replication may fail due to bacterial defense mechanisms like restriction-modification systems [31], abortive infection [32] or the CRISPR-CAS-system [33]. Phages P22 and 9NA use $\mathrm{O}$-antigen as a primary receptor, but other membrane receptors are also necessary for infection $[14,16]$. Their tailspikes' binding and endorhamnosidase function alone may therefore not be sufficient for infection as shown in this work and in earlier studies [21]. However, we observed a positive correlation between TSP O-antigen binding and plaque formation for bacteriophages 9NA and P22 stressing that O-antigen adsorption is necessary for infection. Heterogeneous cell populations with varying $\mathrm{O}$-antigen structure might also affect plating efficiencies. This was observed with phage 9NA that probably only infected one subpopulation in the biphasic strain of S. Paratyphi B 2924 (cf. Table 2). However, the bacterial phenotypes resulting from phase variation may include many more changes on the cellular level beyond $\mathrm{O}$-antigen modification that could interfere with plaque formation.

Taken together, their high O-antigen specificity makes TSP interesting serotyping tools, especially when compared to antibodies. Epitopes recognized by antibodies usually are small and often only span less than one biological repeat of the O-antigen [34]. As a result, typing antibodies cannot always detect $\mathrm{O}$-antigen heterogeneities, in contrast to TSP that have large binding sites spanning at least a complete repeat unit. Moreover, typing antibodies often show batch to batch variations and the agglutination procedure only yields positive or negative results, but no further information on heterogeneous phenotypes in a culture. These drawbacks could be overcome in future by developing a set of TSP with specificities addressing precisely defined bacterial surface structures.

\section{Conclusions}

In this work, we have developed a rapid test of O-antigen composition in Salmonella using bacteriophage tailspike proteins (TSP) as probes, the ELISA-like tailspike adsorption (ELITA) assay. In a microtiter plate format, the ELITA assay could specifically screen which $\mathrm{O}$-antigen serotype is present on cells. TSP are also applicable in culture monitoring by flow cytometry. In contrast to classical type antibodies, TSP could detect phase variable glucosylations on Salmonella O-antigens. TSP have large $\mathrm{O}$-antigen binding sites with high binding specificity, moreover, they are highly protease resistant and thermostable proteins. In cases where the $\mathrm{O}$-antigen specificities of TSP are carefully investigated and therefore their binding behavior is exactly defined, we propose to use TSP as robust serotyping proteins in addition to type antibodies and for monitoring $\mathrm{O}$-antigen phase variations in biotechnology applications.

\section{Methods \\ Materials}

Lipopolysaccharides (LPS), polysaccharides and oligosaccharides were prepared according to the literature $[14,35]$. Phosphate buffered saline (PBS): $16 \mathrm{mM}$ $\mathrm{Na}_{2} \mathrm{HPO}_{4}, 4 \mathrm{mM} \mathrm{KH} \mathrm{PO}_{4}, 115 \mathrm{mM} \mathrm{NaCl}, \mathrm{pH}$ 7.6. TECbuffer: $50 \mathrm{mM}$ Tris/ $\mathrm{HCl}, 5 \mathrm{mM}$ ethylenediaminetetraacetic acid (EDTA), pH 7.6. PBS-T: PBS buffer supplemented 
with $200 \mathrm{mM} \mathrm{NaCl}$ and $0,2 \%(\mathrm{v} / \mathrm{v})$ Tween 20. Flow cytometry (FC) buffer: PBS buffer supplemented with $0.2 \%(\mathrm{w} / \mathrm{v})$ bovine serum albumin (BSA). All chemicals were of analytical grade, and ultrapure water was used throughout.

\section{Tailspike proteins}

To N-terminally fuse Strep-tag'II (IBA, Göttingen, Germany), TSP genes were excised from plasmids $[16,36]$ with restriction enzymes XhoI and BstBI (9NATSP) and NcoI and HindIII (P22TSP) and the fragments ligated into the plasmid pPR-IBA102 (IBA, Göttingen, Germany) and sequenced (GATC Biotech AG, Konstanz, Germany). Expression and purification of 9NA and P22 tailspike proteins (TSP) and mutants followed standard protocols $[16,36]$.

\section{Bacterial strains}

To initially set up the ELITA-assay, $S$. Typhimurium DB7136 LT2 [37] was used, E. coli H TD2158 [38] served as false positive control. All other strains were from the stock culture collection of the National Reference Centre for Salmonellae and other Enterics at the Robert Koch Institute, Wernigerode, Germany, and are listed in Table 3.

\section{ELISA-like tailspike adsorption (ELITA) assay}

Bacteria were cultivated in LB-medium at $37{ }^{\circ} \mathrm{C}$ to OD 0.7-1.3. Bacterial suspensions were washed twice with PBS, diluted to OD 0.2 with PBS and adsorbed to multiwell plates over night at $4{ }^{\circ} \mathrm{C}$. Adsorbed bacteria were inactivated with $2 \%(\mathrm{w} / \mathrm{v})$ phenol in PBS for $20 \mathrm{~min}$. After three wash steps, surfaces were saturated with $2 \%$ $(w / v)$ BSA in PBS. Adsorbed bacteria were incubated with $0.95 \mu \mathrm{g}$ per well of Strep-tag ${ }^{\circ}$ II-TSP in TEC-buffer for 15 min. After washing with PBS-T, bound TSP were detected by adding $0.1 \mu \mathrm{g} \mathrm{ml}^{-1}$ Strep-Tactin ${ }^{\circ}$ labeled with horseradish peroxidase (IBA, Göttingen, Germany), washed and developed with $1 \mathrm{mg} \mathrm{ml}^{-1}$ (w/v) O-Phenylenediamine in $50 \mathrm{mM}$ sodium citrate buffer $(\mathrm{pH} \mathrm{6)}$ containing $0.03 \%$ (v/v) $\mathrm{H}_{2} \mathrm{O}_{2}$ for $5 \mathrm{~min}$. The reaction was stopped with $2 \mathrm{M}$ sulfuric acid and the absorbance at $492 \mathrm{~nm}$ was measured.

\section{Matrix-assisted laser-desorption ionization mass spectrometry (MALDI-MS)}

Samples were mixed in equal volumes with matrix containing $100 \mathrm{mg} \mathrm{ml}^{-1}$ (w/v) 2,5-dihydroxybenzoic acid in 1:1:0.02 (v/v/v) water/acetonitrile/dimethylaniline [39]. Mass spectra were collected on a BrukerMicroflex (Bruker Daltonics, Bremen, Germany) and evaluated with the software mMass [40].
Table 3 Salmonella enterica subsp. enterica strains from the Wernigerode collection used in this study

\begin{tabular}{|c|c|c|c|c|}
\hline \multirow[t]{2}{*}{ Strain } & & \multicolumn{3}{|l|}{ Serotype } \\
\hline & & $\bar{O}$ & $\mathrm{H} 1$ & $\mathrm{H} 2$ \\
\hline $13-02615$ & Paratyphi A & 2 & $a$ & - \\
\hline $13-02749$ & Paratyphi A & 2 & a & - \\
\hline $13-02369$ & Paratyphi A & 1,2 & a & - \\
\hline $13-02623$ & Agona & 4 & $f, g, s$ & - \\
\hline $13-01155$ & Brandenburg & 4 & $\mathrm{I}, \mathrm{V}$ & $e, n, z 15$ \\
\hline $13-03007$ & Brandenburg & 4 & $\mathrm{I}, \mathrm{V}$ & $e, n, z 15$ \\
\hline $13-02696$ & Brandenburg & 4 & $\mathrm{I}, \mathrm{V}$ & $e, n, z 15$ \\
\hline $13-01714$ & Bredeney & 4 & $\mathrm{I}, \mathrm{V}$ & 1,7 \\
\hline $13-03023$ & Brancaster & 4 & z29 & - \\
\hline $13-02636$ & Chester & 4 & $e, h$ & $e, n, x$ \\
\hline $13-03043$ & Derby & 4 & $f, g$ & - \\
\hline $13-00586$ & Heidelberg & 4 & r & 1,2 \\
\hline $13-03022$ & Indiana & 4 & z & 1,7 \\
\hline $13-02835$ & Kalamu & 4 & $\mathrm{z} 4, \mathrm{z} 24$ & - \\
\hline $13-01872$ & Paratyphi B & 4 & $b$ & 1,2 \\
\hline $13-02924$ & Paratyphi B & 4 & $b$ & 1,2 \\
\hline $13-01225$ & Typhimurium & 4 & i & - \\
\hline $13-02608$ & Typhimurium & 4 & i & - \\
\hline $13-02411$ & Wien & 4 & $b$ & $\mathrm{l}, \mathrm{W}$ \\
\hline $13-00008$ & Abony & 4,5 & $b$ & $e, n, x$ \\
\hline 13-00308 & Heidelberg & 4,5 & r & 1,2 \\
\hline 13-01093 & Paratyphi B & 4,5 & $b$ & 1,2 \\
\hline $13-01583$ & Paratyphi B & 4,5 & $b$ & 1,2 \\
\hline $13-02022$ & Paratyphi B & 4,5 & $b$ & 1,2 \\
\hline 06-01900 & Typhimurium & 4,5 & - & - \\
\hline $13-02786$ & Typhimurium & 5 & i & 1,2 \\
\hline $13-03014$ & Enteritidis & 9 & $g, m$ & \\
\hline 13-02939 & Eastbourne & 9 & $e, h$ & 1,5 \\
\hline 08-01911 & Gallinarium & 9 & - & - \\
\hline $13-02970$ & Enteritidis & 9 & $g, m$ & - \\
\hline 13-02971 & Enteritidis & 9 & $g, m$ & - \\
\hline 13-00799 & subspecies II & 9 & $\mathrm{I}, \mathrm{W}$ & $e, n, x$ \\
\hline 13-03072 & Enteritidis & 9,12 & $g, m$ & - \\
\hline 564 & subspecies II & $1,9,12,46,27$ & I,z13,z28 & z39 \\
\hline 565 & subspecies II & $1,9,12,46,27$ & y & z39 \\
\hline $13-00188$ & Anatum & 3,10 & e,h & 1,6 \\
\hline $13-00661$ & Anatum & 3,10 & $e, h$ & 1,6 \\
\hline $11-07456$ & Stendal & 11 & $\mathrm{I}, \mathrm{V}$ & 1,2 \\
\hline $12-05093$ & Rubislaw & 11 & r & $e, n, x$ \\
\hline $\mathrm{T} 4$ & Typhi & $12_{1}, 12_{2}, 12_{3}$ & - & - \\
\hline $\mathrm{T} 2$ & Typhi & $12_{1}, 12_{3}$ & - & - \\
\hline
\end{tabular}




\section{Capillary electrophoresis}

$125 \mu \mathrm{g}$ LPS were incubated with $20 \mu \mathrm{g} \mathrm{mL}^{-1} \mathrm{TSP}$ in $10 \mathrm{mM}$ ammonium acetate over night at $37{ }^{\circ} \mathrm{C}$. After precipitation with $90 \%(\mathrm{v} / \mathrm{v})$ ethanol the supernatant was collected, dried and dissolved in $1.5 \mu \mathrm{L} 8$-aminopyrene1,3,6-trisulfonic acid (200 mM in $15 \%(\mathrm{v} / \mathrm{v})$ acetic acid) and $1.5 \mu \mathrm{L}$ sodium cyanoborohydride $(1 \mathrm{M}$ in tetrahydrofuran), incubated over night at $37{ }^{\circ} \mathrm{C}$ and diluted with $97 \mu \mathrm{L}$ water. Samples were diluted 400 -fold and subjected to capillary electrophoresis on a PA-800 (Beckman Coulter) equipped with a LIF detector as described elsewhere [41].

\section{Flow cytometry}

Bacteria were cultivated in $\mathrm{LB}$ medium at $37{ }^{\circ} \mathrm{C}$ to $\mathrm{OD}$ $0.7-1.3$, inactivated with $1 \%(\mathrm{w} / \mathrm{v})$ phenol and washed with PBS - T. After dilution to OD 0.2 samples were incubated with $5 \mu \mathrm{g} \mathrm{ml}^{-1}$ Strep-tag ${ }^{\circ}$ II-TSP in PBS for $30 \mathrm{~min}$. After washing with PBS-T, cells were stained with $12.5 \mu \mathrm{M}$ SytoRed and $125 \mathrm{ng}$ Strep-Tactin ${ }^{\circ}$, conjugated to Chromeo 488 (IBA, Göttingen, Germany) in PBS with $0.5 \%$ BSA for one hour and washed. All flow cytometry measurements were carried out on a FACS Calibur instrument equipped with the FlowJo data evaluation software (BD Biosciences, Heidelberg, Germany). Samples were diluted until less than 1000 events per min occurred on the fluorescence channel $\left(\lambda_{\mathrm{ex}}: 488 \mathrm{~nm} / \lambda_{\mathrm{em}}: 530 \mathrm{~nm}\right) .2 \times 10^{4}$ events per sample in the side scatter channel were collected.

\section{Bacteriophage assays}

Phages were propagated on S. Typhimurium DB7136 as described and particles quantified as plaque forming units (pfu) $[14,16]$. To determine the number of pfu, bacteria were grown to early log phase in Luria-Bertani (LB) medium at $37^{\circ} \mathrm{C}$, diluted $10^{5}$ fold in soft agar, inoculated with phage suspensions and spread on LB agar plates. Plaque numbers at different phage dilutions $\left(10^{4}-10^{12}\right)$ were counted after incubation at $37^{\circ} \mathrm{C}$ overnight.

\section{Additional files}

Additional file 1: Figure S1. HPAEC-PAD analysis of monosaccharide composition of S. Brancaster. (PDF $106 \mathrm{~kb}$ )

Additional file 2: Figure S2. ELITA assay with 44 Salmonella strains using 9NA and P22TSP as probes. (PDF $194 \mathrm{~kb}$ )

Additional file 3: Figure S3. Full flow cytometry data sets of a representative set of Salmonella strains from serogroup 04,(5) probed with fluorescently tagged TSP. (PDF $1929 \mathrm{~kb}$ )

\section{Acknowledgements}

We thank Stephanie Zimmermann for assistance with flow cytometry and Jörg Fettke for support in CE-LIF analysis.

\section{Funding}

AS was supported by the Leibniz Gemeinschaft in the Leibniz Graduate School of Molecular Biophysics. This work was supported by a grant from the Deutsche Forschungsgemeinschaft [grant number BA 4046/1-2] to SB.

\section{Availability of data and material}

All data generated or analysed during this study are included in this published article and its supplementary information files.

\begin{abstract}
Authors' contributions
AS, WR and SB designed the experiments. AS developed the ELITA test and performed flow cytometry, HPAEC-PAD and CE-LIF and evaluated all data. WR provided the strain collection. NKB carried out the phage plating assays. AS and SB drafted the manuscript. All authors read and approved the final manuscript.
\end{abstract}

\section{Competing interests}

The authors declare that they have no competing interests.

\section{Consent for publication}

Not applicable.

Ethics approval and consent to participate

Not applicable.

\section{Author details}

${ }^{1}$ Physikalische Biochemie, Universität Potsdam, Karl-Liebknecht-Str. 24-25, Golm 14476, Germany. ${ }^{2}$ National Reference Centre for Salmonella and other Bacterial Enterics, Robert Koch Institute, Burgstraße 37, Wernigerode 38855, Germany.

Received: 15 October 2015 Accepted: 31 August 2016

Published online: 07 September 2016

\section{References}

1. Pitout JDD, Laupland KB. Extended-spectrum beta-lactamase-producing enterobacteriaceae: an emerging public-health concern. Lancet Infect Dis. 2008;8(3):159-66.

2. Viertel TM, Ritter K, Horz H-P. Viruses versus bacteria-novel approaches to phage therapy as a tool against multidrug-resistant pathogens. J Antimicrob Chemoth. 2014;69(9):2326-36.

3. Grimont P, Weill F. Antigenic formulae of the Salmonella serovars. 9th ed. Geneva: WHO Collaborating Centre for Reference and Research on Salmonella; 2007.

4. Raetz CR, Whitfield C. Lipopolysaccharide endotoxins. Annu Rev Biochem. 2002;71:635-700.

5. Bogomolnaya LM, Santiviago CA, Yang H-J, Baumler AJ, Andrews-Polymenis HL. 'Form variation' of the 012 antigen is critical for persistence of Salmonella Typhimurium in the murine intestine. Mol Microbiol. 2008;70(5):1105-19.

6. Broadbent SE, Davies MR, van der Woude MW. Phase variation controls expression of Salmonella lipopolysaccharide modification genes by a DNA methylation-dependent mechanism. Mol Microbiol. 2010;77(2):337-53.

7. Davies MR, Broadbent SE, Harris SR, Thomson NR, van der Woude MW. Horizontally Acquired Glycosyltransferase Operons Drive Salmonellae Lipopolysaccharide Diversity. PLoS Genet. 2013;9(6):e1003568.

8. Nikaido H, Nikaido K, Nakae T, Makela PH. Glucosylation of lipopolysaccharide in Salmonella - Biosynthesis of O antigen factor 12 2. 1. Over-all reaction. J Biol Chem. 1971;246(12):3902-11.

9. Hellerqvist CG, Lindberg B, Svensson S, Holme T, Lindberg AA. Structural studies on O-specific side-chains of cell-wall lipopolysaccharide from Salmonella Typhimurium 395 MS. Carbohyd Res. 1968;8(1):43-55.

10. Alt K, Simon S, Helmeke C, Kohlstock C, Prager R, Tietze E, Rabsch W, Karagiannis I, Werber D, Frank C, et al. Outbreak of Uncommon O4 Non-Agglutinating Salmonella Typhimurium Linked to Minced Pork, Saxony-Anhalt, Germany, January to April 2013. Plos One. 2015;10(6):e0128349.

11. Zanoni I, Bodio C, Broggi A, Ostuni R, Caccia M, Collini M, Venkatesh A, Spreafico R, Capuano G, Granucci F. Similarities and differences of innate immune responses elicited by smooth and rough LPS. Immunol Lett. 2012;142(1-2):41-7. 
12. Kim M, Ryu S. Spontaneous and transient defence against bacteriophage by phase-variable glucosylation of O-antigen in Salmonella enterica serovar Typhimurium. Mol Microbiol. 2012;86(2):411-25.

13. Kintz E, Davies MR, Hammarlof DL, Canals R, Hinton JCD, van der Woude MW. A BTP1 prophage gene present in invasive non-typhoidal Salmonella determines composition and length of the O-antigen of the lipopolysaccharide. Mol Microbiol. 2015;96(2):263-75.

14. Andres D, Baxa U, Hanke C, Seckler R, Barbirz S. Carbohydrate binding of Salmonella phage P22 tailspike protein and its role during host cell infection. Biochem Soc Trans. 2010;038(5):1386-9.

15. Andres D, Hanke C, Baxa U, Seul A, Barbirz S, Seckler R. Tailspike interactions with lipopolysaccharide effect DNA ejection from phage P22 particles in vitro. J Biol Chem. 2010;285(47):36768-75.

16. Andres D, Roske Y, Doering C, Heinemann U, Seckler R, Barbirz S. Tail morphology controls DNA release in two Salmonella phages with one lipopolysaccharide receptor recognition system. Mol Microbiol. 2012;83(6):1244-53.

17. Andres D, Gohlke U, Broeker NK, Schulze S, Rabsch W, Heinemann U, Barbirz S, Seckler R. An essential serotype recognition pocket on phage P22 tailspike protein forces Salmonella enterica serovar Paratyphi A O-antigen fragments to bind as nonsolution conformers. Glycobiology. 2013;23(4):486-94.

18. Broeker NK, Gohlke U, Müller JJ, Uetrecht C, Heinemann U, Seckler R, Barbirz S. Single amino acid exchange in bacteriophage HK620 tailspike protein results in thousand-fold increase of its oligosaccharide affinity. Glycobiology. 2013;23(1):59-68.

19. Baxa U, Cooper A, Weintraub A, Pfeil W, Seckler R. Enthalpic barriers to the hydrophobic binding of oligosaccharides to phage P22 tailspike protein. Biochemistry. 2001:40(17):5144-50.

20. Barbirz S, Becker M, Freiberg A, Seckler R. Phage Tailspike Proteins with beta-Solenoid Fold as Thermostable Carbohydrate Binding Materials. Macromol Biosci. 2009;9(2):169-73.

21. Wollin R, Eriksson U, Lindberg AA. Salmonella bacteriophage glycanases: endorhamnosidase activity of bacteriophages P27, 9NA, and KB1. J Virol. 1981;38(3):1025-33.

22. Hellerqvist CG, Lindberg B, Svensson S, Holme T, Lindberg AA. Structural studies on O-specific side-Chains of cell-wall lipopolysaccharide from Salmonella Typhimurium LT2. Carbohyd Res. 1969:9(2):237-41.

23. Davey HM, Winson MK. Using flow cytometry to quantify microbial heterogeneity. Curr Issues Mol Biol. 2003;5(1):9-15.

24. Chaiet $L$, Wolf FJ. Properties of streptavidin biotin-binding protein produced by Streptomycetes. Arch Biochem Biophys. 1964;106(1-3):1-5.

25. Makela PH. Glucosylation of lipopolysaccharide in Salmonella: mutants negative for $O$ antigen factor 1221. J Bacteriol. 1973;116(2):847-56.

26. Steinbacher S, Baxa U, Miller S, Weintraub A, Seckler R, Huber R. Crystal structure of phage P22 tailspike protein complexed with Salmonella sp. O-antigen receptors. Proc Natl Acad Sci U S A. 1996;93(20):10584-8.

27. Ogunremi D, Kelly H, Dupras AA, Belanger S, Devenish J. Development of a New Molecular Subtyping Tool for Salmonella enterica Serovar Enteritidis Based on Single Nucleotide Polymorphism Genotyping Using PCR. J Clin Microbiol. 2014;52(12):4275-85.

28. Rondini S, Micoli F, Lanzilao L, Gavini M, Alfini R, Brandt C, Clare S, Mastroeni P, Saul A, MacLennan CA. Design of Glycoconjugate Vaccines against Invasive African Salmonella enterica Serovar Typhimurium. Infect Immun. 2015;83(3):996-1007.

29. Meloni E, Colucci AM, Micoli F, Sollai L, Gavini M, Saul A, Di Cioccio V, MacLennan CA. Simplified low-cost production of O-antigen from Salmonella Typhimurium Generalized Modules for Membrane Antigens (GMMA). J Biotechnol. 2015;198:46-52.

30. Rabsch W, Truepschuch S, Windhorst D, Gerlach RG. Typing phages and prophages of Salmonella. In: Porwollik S, editor. Salmonella: From Genome to Function. Norfolk: Caister Academic Press; 2011. p. 25-48.

31. Loenen WAM, Dryden DTF, Raleigh EA, Wilson GG, Murray NE. Highlights of the DNA cutters: a short history of the restriction enzymes. Nucleic Acids Res. 2014;42(1):3-19.

32. Dy RL, Przybilski R, Semeijn K, Salmond GPC, Fineran PC. A widespread bacteriophage abortive infection system functions through a Type IV toxin-antitoxin mechanism. Nucleic Acids Res. 2014;42(7):4590-605.

33. Hynes AP, Villion M, Moineau S. Adaptation in bacterial CRISPR-Cas immunity can be driven by defective phages. Nat Commun. 2014;5. http:// www.nature.com/ncomms/2014/140724/ncomms5399/full/ncomms5399. html.
34. Bundle DR, Eichler E, Gidney MA, Meldal M, Ragauskas A, Sigurskjold BW, Sinnott B, Watson DC, Yaguchi M, Young NM. Molecular recognition of a Salmonella trisaccharide epitope by monoclonal antibody Se155-4. Biochemistry. 1994;33(17):5172-82.

35. Zaccheus MV, Broeker NK, Lundborg M, Uetrecht C, Barbirz S, Widmalm G. Structural studies of the O-antigen polysaccharide from Escherichia coli TD2158 having 018 serogroup specificity and aspects of its interaction with the tailspike endoglycosidase of the infecting bacteriophage HK620. Carbohydr Res. 2012;357:118-25.

36. Baxa U, Steinbacher S, Miller S, Weintraub A, Huber R, Seckler R. Interactions of phage P22 tails with their cellular receptor, Salmonella O-antigen polysaccharide. Biophys J. 1996;71(4):2040-8.

37. Winston F, Botstein D, Miller JH. Characterization of amber and ochre suppressors in Salmonella typhimurium. J Bacteriol. 1979;137(1):433-9.

38. Dhillon TS, Poon AP, Chan D, Clark AJ. General transducing phages like Salmonella phage P22 isolated using a smooth strain of Escherichia coli as host. FEMS Microbiol Lett. 1998;161(1):129-33.

39. Ropartz D, Bodet P-E, Przybylski C, Gonnet F, Daniel R, Fer M, Helbert W, Bertrand D, Rogniaux H. Performance evaluation on a wide set of matrix-assisted laser desorption ionization matrices for the detection of oligosaccharides in a high-throughput mass spectrometric screening of carbohydrate depolymerizing enzymes. Rapid Commun Mass Sp. 2011:25(14):2059-70.

40. Strohalm M, Kavan D, Novak P, Volny M, Havlicek V. mMass 3: A CrossPlatform Software Environment for Precise Analysis of Mass Spectrometric Data. Anal Chem. 2010;82(11):4648-51.

41. Malinova I, Mahlow S, Alseekh S, Orawetz T, Fernie AR, Baumann O, Steup M, Fettke J. Double Knockout Mutants of Arabidopsis Grown under Normal Conditions Reveal that the Plastidial Phosphorylase Isozyme Participates in Transitory Starch Metabolism. Plant Physiol. 2014;164(2):907-21.

42. Varki A, Sharon N. Symbolic representations of monosaccharides and linkages. In: Varki A, Cummings RD, Esko JD, editors. Essentials of Glycobiology. 2nd ed. Cold Spring Harbor: Cold Spring Harbor Laboratory Press; 2009.

43. Schroedinger LLC. The PyMOL Molecular Graphics System, Version 1.7.6. 2015.

44. Eriksson U, Svenson SB, Lonngren J, Lindberg AA. Salmonella phage glycanases: substrate specificity of the phage P22 endo-rhamnosidase. J Gen Virol. 1979;43(3):503-11

\section{Submit your next manuscript to BioMed Central and we will help you at every step:}

- We accept pre-submission inquiries

- Our selector tool helps you to find the most relevant journal

- We provide round the clock customer support

- Convenient online submission

- Thorough peer review

- Inclusion in PubMed and all major indexing services

- Maximum visibility for your research

Submit your manuscript at www.biomedcentral.com/submit
Biomed Central 